\title{
Designing the Game to Play: Optimizing Payoff Structure in Security Games
}

\author{
Zheyuan Ryan Shi ${ }^{1 *}$, Ziye Tang ${ }^{2 *}$, Long Tran-Thanh ${ }^{3}$, Rohit Singh $^{4}$, Fei Fang ${ }^{2}$ \\ ${ }^{1}$ Swarthmore College, USA \\ ${ }^{2}$ Carnegie Mellon University, USA \\ ${ }^{3}$ University of Southampton, UK \\ ${ }^{4}$ World Wide Fund for Nature, Cambodia
}

\begin{abstract}
We study Stackelberg Security Games where the defender, in addition to allocating defensive resources to protect targets from the attacker, can strategically manipulate the attacker's payoff under budget constraints in weighted $L^{p}$-norm form regarding the amount of change. For the case of weighted $L^{1}$-norm constraint, we present (i) a mixed integer linear program-based algorithm with approximation guarantee; (ii) a branch-and-bound based algorithm with improved efficiency achieved by effective pruning; (iii) a polynomial time approximation scheme for a special but practical class of problems. In addition, we show that problems under budget constraints in $L^{0}$ and weighted $L^{\infty}$ norm form can be solved in polynomial time.
\end{abstract}

\section{Introduction}

Research efforts in security games have led to success in various domains, ranging from protecting critical infrastructure [Letchford and Conitzer, 2013; Wang et al., 2016] and catching fare invaders in metro systems [Yin et al., 2012], to combating poaching [Fang et al., 2016] and preventing cyber intrusions [Durkota et al., 2015; Basilico et al., 2016]. In these games, a defender protects a set of targets from an attacker by allocating defensive resources. One key element that characterizes the strategies of the players is the payoff structure. Existing work in this area typically treats the payoff structure of the players as given parameters, sometimes with uncertainties known a priori given the nature of the domain. However, under various circumstances, the defender is able to change the attacker's payoff, thus rendering the existing models inadequate in expressiveness. For example, in wildlife poaching, the law enforcement agency may charge a variable fine if the poacher is caught at different locations, e.g., in the core area vs. in the non-core area. In cybersecurity, the network administrator may change the actual or appeared value of any network node for a potential attacker. In these cases, the defender's decision making is two-staged: she chooses the payoff structure, as well as the strategy of allocating defensive resources. With a properly chosen payoff structure, the defender may save the effort by achieving much better utility with the same or even less amount of resources.

\footnotetext{
${ }^{*}$ Z. R. Shi and Z. Tang contributed equally to this work.
}

As existing work in security games does not provide adequate tools to deal with this problem (see Section 2 for more details), we aim to fill this gap as follows. We study how to design the attacker's payoff structure in security games given budget constraints in weighted $L^{p}$-norm $(p=0,1, \infty)$. That is, the distance between the original payoff structure and the modified payoff structure is bounded, using distance metrics such as Manhattan distance (i.e., $L^{1}$-norm) with varying weights for reward and penalty of different targets. The intuition behind this setting is that the defender can change the payoffs to make a target that is preferable to the defender more attractive to the attacker and disincentivize the attacker from attacking targets that can lead to a significant loss to the defender. More change incurs a higher cost to the defender and the defender has a fixed budget for making the changes. Our findings can be summarized as follows:

$L^{1}$-norm case: When the budget constraint is in weighted $L^{1}$-norm form, i.e. additive cost, our contribution is threefold. (i) We exploit several key properties of the optimal manipulation and propose a mixed integer linear program (MILP)based algorithm with approximation guarantee. (ii) We propose a novel branch-and-bound approach with improved efficiency achieved by effective pruning for the general case. (iii) Finally, we show that a polynomial time approximation scheme (PTAS) exists for a special but practical case where the budget is very limited and the manipulation cost is uniform across targets. The PTAS is built upon the key observation that there is an optimal solution where no more than two targets' payoffs are changed in this restricted case.

$L^{0}$ and $L^{\infty}$-norm cases: We propose $a O\left(n^{3}\right)$ and $a$ $O\left(n^{2} \log n\right)$ algorithm for problems under budget constraints in $L^{0}$-norm form and weighted $L^{\infty}$-norm form, respectively, where $n$ is the total number of targets. For $L^{0}$-norm form budget, i.e. limited number of targets to manipulate, our algorithm converts the problem into $O\left(n^{2}\right)$ subproblems and reduces each subproblem to a problem of finding a subset of items with the maximum average weight. The latter can be solved in $O(n)$ time. For $L^{\infty}$-norm form budget, i.e. limited range of manipulation on each target, we reduce the problem to traditional Stackelberg Security Games with fixed payoff structure, which again admits an efficient algorithm.

Numerical evaluation: We provide extensive experimental evaluation for the proposed algorithms. For problems with $L^{1}$-norm form budget constraint, we show that the branchand-bound approach with an additive approximation guaran- 
tee can solve up to hundreds of targets in a few minutes. This is faster than other baseline algorithms we compare to. Somewhat surprisingly, naively solving $n$ non-convex subproblems using interior point method achieves good performance in practice despite that there is no theoretical guarantee of solution quality. We also evaluate the proposed $O\left(n^{3}\right)$ algorithm for the $L^{0}$-norm form case and show its superior performance over two greedy algorithms and a MILP based algorithm.

\section{Preliminaries and Related Work}

The security game that we consider in this paper features a set of $n$ targets, $T=\{1,2, \ldots, n\}$. The defender has $r$ units of defensive resources, each can protect one target. The attacker can choose to attack one target after observing the defender's strategy. If the defender covers target $i$ when it is attacked, the defender gets a reward $R_{i}^{d} \geq 0$ and the attacker gets a penalty $P_{i}^{a} \leq 0$. Otherwise, the defender gets a penalty $P_{i}^{d} \leq 0$ and the attacker gets a reward $R_{i}^{a} \geq 0$. When the defender commits to a mixed strategy $c$, that is, covering target $i$ with probability $c_{i}$, the defender's and attacker's expected utilities when target $i$ is attacked are $U_{i}^{d}=c_{i} R_{i}^{d}+\left(1-c_{i}\right) P_{i}^{d}$ and $U_{i}^{a}=c_{i} P_{i}^{a}+\left(1-c_{i}\right) R_{i}^{a}$, respectively.

We adopt the commonly used solution concept of Strong Stackelberg Equilibrium (SSE) [Kiekintveld et al., 2009]. At an SSE, the defender chooses an optimal strategy that leads to the highest expected utility for her when the attacker chooses a best response (assumed to be a pure strategy w.l.o.g), breaking ties in favor of the defender. Given a coverage $c$, the attack set $\Gamma \subseteq T$ contains all the targets which have a weakly higher attacker's expected utility than any other target, i.e.,

$$
\Gamma=\left\{j \in T: U_{j}^{a} \geq U_{k}^{a}, \forall k \in T\right\}
$$

[Kiekintveld et al., 2009] show that there exists an SSE where the defender only covers the targets in the attack set.

Given the game parameters, the optimal defender strategy in such a game can be computed using multiple linear programs (LPs) [Conitzer and Sandholm, 2006] or an efficient $O\left(n^{2}\right)$ algorithm called ORIGAMI [Kiekintveld et al., 2009] based on enumerating the possible attack sets. We leverage insights from both works to devise our algorithms.

Although many algorithms have been developed for security games under various settings, in most of the existing literature, the payoff structure is treated as fixed and cannot be changed by the defender, either in the full information case [Korzhyk et al., 2010; Paruchuri et al., 2008; Laszka et al., 2017], or in the presence of payoff uncertainties [Kiekintveld et al., 2013; 2011; Yin and Tambe, 2012; Letchford et al., 2009; Blum et al., 2014]. As mentioned earlier, in many real-world scenarios the defender has control over the attacker's payoffs. The approaches above ignore this aspect and thus leave room for further optimization.

Indeed, despite its significance, jointly optimizing the payoff structure and the resource allocation is yet underexplored. A notable exception, and a most directly related work to ours, is the audit game model [Blocki et al., 2013; 2015]. The defender can choose target-specific "punishment rates", in order to maximize her expected utility offset by the cost of setting the punishment rate. Compared with their model, ours is more general in that we allow not only manipulation of attacker's penalty, but also attacker's reward.
This realistic extension makes their core techniques inapplicable. Also, we treat the manipulation cost as a constraint instead of a regularization term in the objective function, for in some real-world settings, payoffs can be manipulated only once, yet the defender may face multiple attacks afterwards. This makes it hard to determine the regularization coefficient. Another closely related work [Schlenker et al., 2018] focuses on the use of honeypot [Kiekintveld et al., 2015; Durkota et al., 2015; Píbil et al., 2012]. It studies the problem of deceiving a cyber attacker by manipulating the attacker's (believed) payoff. However, it assumes the defender can only change the payoff structure, ignoring the allocation of defensive resources after the manipulation. [Horák et al., 2017] study the manipulation of attacker's belief in a repeated game. They assume actively engaging attacker and defender, which is not the case in our problem.

If we conceptually decouple the payoff manipulation from resource allocation, the defender faces a two-stage decision. She first chooses the structure of the game, and then plays the game. Thus, our problem may be viewed as a mechanism design problem, albeit not in a conventional setting. Most work in mechanism design considers private information games [Fujishima et al., 1999; Myerson, 1989], while in our work, and in most security game literature, the payoff information is public. Some design the incentive mechanism using a Stackelberg game [Kang and Wu, 2015], with applications to network routing [Sharma and Williamson, 2007], mobile phone sensing [Yang et al., 2012], and ecology surveillance [Xue et al., 2016]. However, these works solve the Stackelberg game to design the mechanism, rather than designing the structure of the Stackelberg game.

\section{Optimizing Payoff with Budget Constraint in Weighted $L^{1}$-norm Form}

In this section, we focus on computing the optimal way of manipulating attacker's payoffs and allocating defensive resources. Payoff manipulation is subject to a budget constraint in weighted $L^{1}$-norm form, i.e., the defender can change the attacker's reward and penalty, at a cost that grows linearly in the amount of change. The cost rate, referred to as weights, may be different across targets. This is an abstraction of several domains. For example, a network administrator may change the actual or appeared value of any network node although such change often incurs time and hardware costs.

Let $R^{a}, P^{a}, \bar{R}^{a}, \bar{P}^{a}$ denote the attacker's reward and penalty vectors before and after the manipulation. Similar to the initial payoff structure, we require that $\bar{R}^{a} \geq 0 \geq \bar{P}^{a}$ and denote $D_{j}=R_{j}^{a}-P_{j}^{a}$. Let $\epsilon=\bar{R}^{a}-R^{a}$ and $\bar{\delta}=\bar{P}^{a}-P^{a}$ be the amount of change in attacker's reward and penalty and $\mu, \theta$ the weights on $\epsilon, \delta$ resp.. The weighted $L^{1}$ budget constraint is then $\sum_{j}\left(\mu_{j}\left|\epsilon_{j}\right|+\theta_{j}\left|\delta_{j}\right|\right) \leq B$ where $B$ is the budget. The defender's strategy is characterized by $(c, \epsilon, \delta)$. Given this strategy, in the manipulated game the attacker attacks some target $t$, which belongs to the attack set $\Gamma$. We first show some properties of the optimal solution.

Property 1. There is an optimal solution $(c, \epsilon, \delta)$ with corresponding attack target $t$ and attack set $\Gamma$ which satisfies the following conditions:

1. $c_{j}=0, \epsilon_{j}=0, \delta_{j}=0, \forall j \notin \Gamma$. 
2. $\epsilon_{t} \geq 0, \delta_{t} \geq 0 ; \epsilon_{j} \leq 0, \delta_{j} \leq 0, \forall j \neq t$.

3. $\delta_{t} \epsilon_{t}\left(\delta_{t}+P_{t}^{a}\right)=0$ and $\delta_{j} \epsilon_{j}\left(R_{j}^{a}+\epsilon_{j}\right)=0, \forall j \neq t$.

Proof sketch. Condition 1: If any $\epsilon_{j}, \delta_{j} \neq 0$ with $j \notin \Gamma$, we may either set $\epsilon_{j}, \delta_{j}=0$ or push $j$ into the attack set. There is no need to protect a target that is not in the attack set. Condition 2: Flipping the sign of $\epsilon$ and $\delta$ leads to no better solution. Condition 3: For each target $i$, we can shift the change on $R_{i}^{a}$ to $P_{i}^{a}$ and vice versa while one is more budget efficient than the other depending on coverage. We can show that these manipulations can be done simultaneously. ${ }^{1}$

Similar to the multiple LPs formulation in [Conitzer and Sandholm, 2006], we consider $n$ subproblems $\mathcal{P}_{i}$, each assuming some target $i \in T$ is the attack target, and the best solution among all $n$ subproblems is the optimal defender strategy. Condition 2 in Property 1 shows it is possible to infer the sign of $\epsilon$ and $\delta$ given the attack target. So in the sequel, we abuse the notation by treating $\epsilon, \delta$ as the absolute value of the amount of change, and assume w.l.o.g. that in $\mathcal{P}_{i}$, $\bar{R}_{i}^{a}=R_{i}^{a}+\epsilon_{i}, \bar{P}_{i}^{a}=P_{i}^{a}+\delta_{i}$ and $\forall j \neq i, \bar{R}_{j}^{a}=R_{j}^{a}-\epsilon_{j}, \bar{P}_{j}^{a}=$ $P_{j}^{a}-\delta_{j}$. Thus, a straightforward formulation for $\mathcal{P}_{i}$ is

$$
\begin{aligned}
\max _{c, \epsilon, \delta} & U_{i}^{d}=R_{i}^{d} c_{i}+P_{i}^{d}\left(1-c_{i}\right)=c_{i} D_{i}+P_{i}^{d} \\
\text { s.t. } & U_{i}^{a}=c_{i}\left(P_{i}^{a}+\delta_{i}\right)+\left(1-c_{i}\right)\left(R_{i}^{a}+\epsilon_{i}\right) \\
& \geq U_{j}^{a}=c_{j}\left(P_{j}^{a}-\delta_{j}\right)+\left(1-c_{j}\right)\left(R_{j}^{a}-\epsilon_{j}\right), \forall j \neq i \\
& \sum_{j}\left(\mu_{j} \epsilon_{j}+\theta_{j} \delta_{j}\right) \leq B \\
& \sum_{j} c_{j} \leq r \\
& R_{j}^{a}-\epsilon_{j} \geq 0, \quad \forall j \neq i \\
& P_{i}^{a}+\delta_{i} \leq 0 \\
& c_{j}, \epsilon_{j}, \delta_{j} \geq 0, \quad c_{j} \leq 1, \quad \forall j \in T
\end{aligned}
$$

The above formulation is non-convex due to the quadratic terms in Constraint 3 which leads to an indefinite Hessian matrix, and thus no existing solvers can guarantee global optimality for the above formulation.

\subsection{A MILP-based Solution with Approximation Guarantee}

To find a defender strategy with solution quality guarantee, we solve the atomic version of the subproblems with MILPs. We show an approximation guarantee which improves as the fineness of discretization grows. We further propose a branch-and-bound-like framework for pruning subproblems to improve runtime efficiency.

In the atomic version of the payoff manipulation problem, we assume the defender can only make atomic changes, with the minimum amount of change given as $\rho_{0}$. We refer to the atomic version of $\mathcal{P}_{i}$ as $\mathcal{A P} \mathcal{P}_{i} \mathcal{A P}_{i}$ can be formulated as the MILP in Equations 9-19. We simplify the objective function as $c_{i}$ since $D_{i} \geq 0$. All constraints involving sub/super-script $j, k$ without a summation apply to all proper range of summation indices. We use binary representation for $\bar{R}_{i}^{a} / \rho_{0}$ and $\bar{P}_{i}^{a} / \rho_{0}$ in constraints $10-14$. The binary representation results

${ }^{1}$ Due to limited space, the full proofs are included in the full version of the paper: https://arxiv.org/abs/1805.01987 in bilinear terms like $y_{j}^{k} c_{j}$. We introduce variables $\alpha_{j}^{k}, \beta_{j}^{k}$ and constraints 18-19 to linearize them.

$$
\max _{y_{j}^{k}, z_{j}^{k}, c_{j}} c_{i}
$$

\section{s.t. Constraint $4-8$}

$$
\begin{aligned}
& \epsilon_{i}=\rho_{0} \sum_{k} 2^{k} y_{i}^{k}-R_{i}^{a} \\
& \epsilon_{j}=R_{j}^{a}-\rho_{0} \sum_{k} 2^{k} y_{j}^{k}, \quad \forall j \neq i \\
& \delta_{i}=-\rho_{0} \sum_{k} 2^{k} z_{i}^{k}-P_{i}^{a} \\
& \delta_{j}=P_{j}^{a}+\rho_{0} \sum_{k} 2^{k} z_{j}^{k}, \quad \forall j \neq i \\
& y_{j}^{k}, z_{j}^{k} \in\{0,1\} \\
& v_{i} \geq v_{j} \\
& v_{i}=R_{i}^{a}+\epsilon_{i}-\rho_{0} \sum_{k} 2^{k}\left(\alpha_{i}^{k}+\beta_{i}^{k}\right) \\
& v_{j}=R_{j}^{a}-\epsilon_{j}-\rho_{0} \sum_{k} 2^{k}\left(\alpha_{j}^{k}+\beta_{j}^{k}\right), \forall j \neq i \\
& 0 \leq \alpha_{j}^{k} \leq y_{j}^{k}, \quad c_{j}-\left(1-y_{j}^{k}\right) \leq \alpha_{j}^{k} \leq c_{j} \\
& 0 \leq \beta_{j}^{k} \leq z_{j}^{k}, \quad c_{j}-\left(1-z_{j}^{k}\right) \leq \beta_{j}^{k} \leq c_{j}
\end{aligned}
$$

The optimal defender strategy for the atomic payoff manipulation problem can be found by checking the solution to all the subproblems and compare the corresponding $U_{i}^{d}$. We can also combine all the subproblems by constructing a single MILP, with additional variables indicating which subproblem is optimal. The details can be found in the full version.

A natural idea to approximate the global optima of the original $L^{1}$-constrained payoff manipulation problem is, for each attack target $i$, approximate $\mathcal{P}_{i}$ with $\mathcal{A P}_{i}$ using small enough $\rho_{0}$. Theorem 1 below shows such an approximation bound.

Theorem 1. The solution of the atomic problem is an additive $\max _{i} \frac{2 \rho_{0}\left(R_{i}^{d}-P_{i}^{d}\right)}{R_{i}^{a}}$-approximation to the original problem.

Proof sketch. The floor and ceiling notations are about the "integral grid" defined by $\rho_{0}$. Suppose $\left(c^{*}, \epsilon^{*}, \delta^{*}\right)$ is an optimal solution to $\mathcal{P}_{i}$. Let $\epsilon^{\prime}=\left\lfloor\epsilon^{*}\right\rfloor, \delta^{\prime}=\left\lfloor\delta^{*}\right\rfloor$, and $c^{\prime}=c^{*}$ except $c_{i}^{\prime}=c_{i}^{*}-2 \rho_{0} /\left(D_{i}+\epsilon_{i}^{\prime}-\delta_{i}^{\prime}\right)$. We can show such feasible solutions yield the desired approximation bound.

We note that the idea of discretizing the manipulation space is similar to [Blocki et al., 2013; 2015]. Yet allowing changes in both reward and penalty and the difference in objective function make our formulation different and the reduction to SOCP used in [Blocki et al., 2015] inapplicable.

We can further improve the practical runtime of the MILPs by pruning and prioritizing subproblems as shown in Alg. 1 . We first compute a global lower bound by checking a sequence of greedy manipulations. Inspired by Condition 2 and 3 in Property 1, we greedily spend all the budget on one target to increase its reward or penalty, leaving all other targets' payoff parameters unchanged (Lines 2 - 8).

Upper bounds in $\mathcal{P}_{i}$ can be computed with budget reuse: we independently spend the full amount of budget $B$ on each target to increase $R_{i}^{a}$ and $P_{i}^{a}$ and decrease $R_{j}^{a}$ and $P_{j}^{a}, j \neq i$, as much as possible. For the ease of notation, in Alg. 1 we 


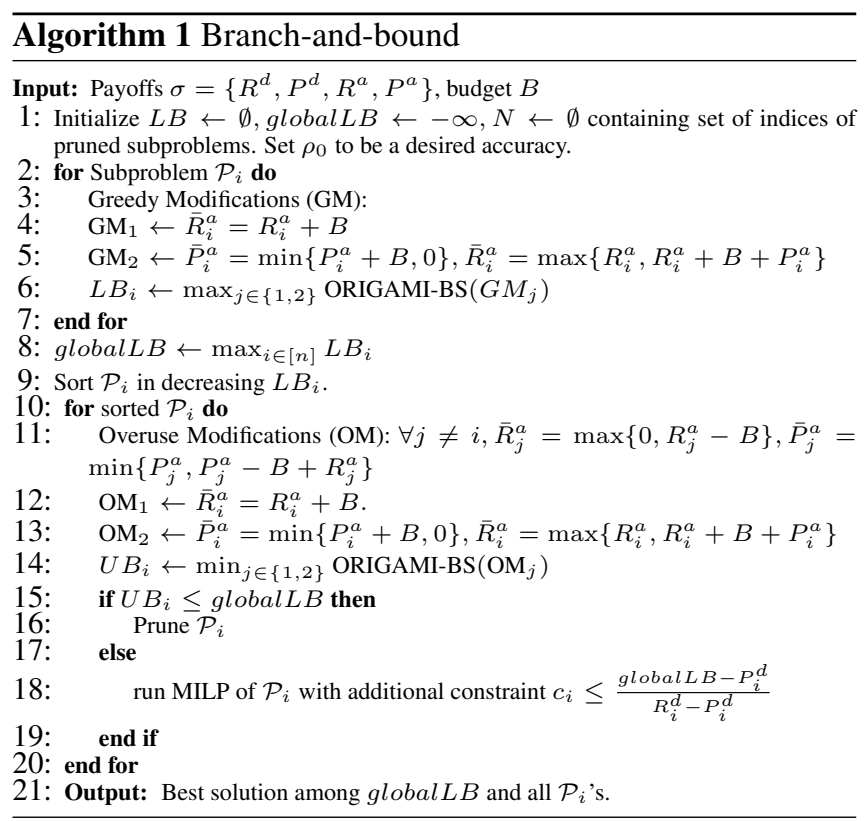

assume manipulations have uniform cost. The weighted case can be easily extended.

The subproblem $\mathcal{P}_{i}$ is pruned if its upper bound is lower than the global lower bound. To make the pruning more efficient, we solve subproblems in descending order of their corresponding lower bounds, hoping for an increase in the global lower bound. For subproblems that cannot be pruned, we set $\rho_{0}$ to the desired accuracy and solve the MILP to approximate the subproblem optima. We also add to the MILP the linear constraint on $c_{i}$ derived from the global lower bound.

To get the bounds, we call an improved version of the ORIGAMI algorithm in [Kiekintveld et al., 2009] by doing a binary search on the size of the attack set $\Gamma$, and solve the linear system. It is denoted as ORIGAMI-BS in Alg. 1. Recall $r$ is the defender's total resource. Let $M$ be the attacker's expected utility for attack set and $\bar{E}_{k}=\frac{1}{R_{i}^{a}-P_{i}^{a}}$. From $U_{i}^{a}=U_{j}^{a}, \forall j \in \Gamma$ and $\sum_{j \in \Gamma} c_{j}=r$, we obtain

$$
\begin{aligned}
M & =\frac{\sum_{k \in \Gamma} \bar{R}_{k}^{a} \bar{E}_{k}-r}{\sum_{k \in \Gamma} \bar{E}_{k}} \\
c_{j} & =\bar{E}_{j}\left(\frac{\sum_{k \in \Gamma}\left(\bar{R}_{j}^{a}-\bar{R}_{k}^{a}\right) \bar{E}_{k}+r}{\sum_{k \in \Gamma} \bar{E}_{k}}\right), \quad \forall j \in \Gamma
\end{aligned}
$$

We iteratively cut the search space by half based on $c_{j}$ and $M$. The complexity improves from $O\left(n^{2}\right)$ to $O(n \log n)$. A complete description can be found in the full version.

We end this subsection by remarking that atomic payoff manipulation arises in many real-world applications. For example, it is infeasible for the wildlife ranger to charge the poacher a fine of $\$ 100 / 3$. In those cases, our proposed MILP formulation could be directly applied.

\subsection{PTAS for Limited Budget and Uniform Costs}

We show that for a special but practical class of problems, there exist a PTAS. In many applications, the defender has only a limited budget $B \leq \min _{j \in T}\left\{\left|P_{j}^{a}\right|, R_{j}^{a}\right\}$. Additionally, the weights on $\epsilon$ and $\delta$ are the same. W.l.o.g., we assume

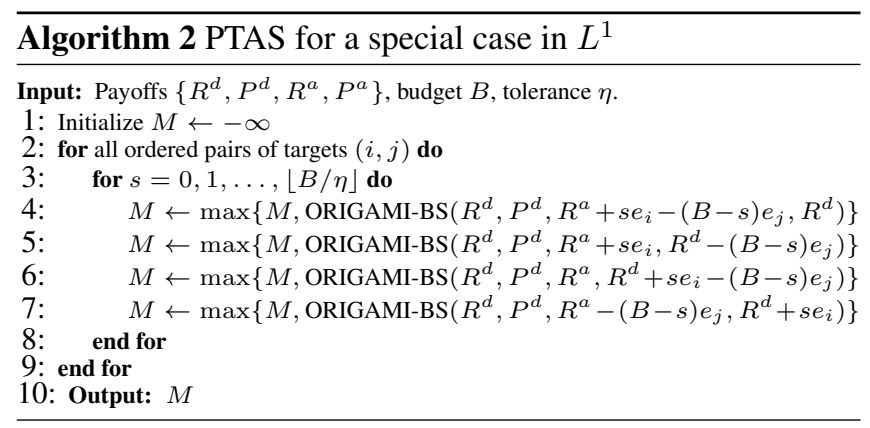

$\mu_{j}=\theta_{j}=1$. We first show a structural theorem below and then discuss its algorithmic implication.

Theorem 2. When budget $B \leq \min _{j \in T}\left\{\left|P_{j}^{a}\right|, R_{j}^{a}\right\}$ and $\mu_{j}=\theta_{j}=1, \forall j \in T$, there exists an optimal solution which manipulates the attack target and at most one other target.

Proof sketch. Since $B$ is limited, either $R_{t}^{a}$ or $P_{t}^{a}$ is unchanged according to Condition 3 of Property 1. Assume all manipulations happen on attacker's reward. If some three targets get manipulated, we can simultaneously increase $\epsilon_{t}$ for attack target $t$ and decrease $\epsilon_{j}$ for $j \neq t$ such that $j$ 's utility increases to be the same as target $t$, until some $\epsilon_{j}$ becomes 0 . After such change, the defender's utility does not decrease, and the number of targets manipulated decreases. Other cases also hold due to symmetry.

The theorem above is tight, i.e. we show in the full version an instance where two targets are manipulated. When $B \leq \min _{j \in T}\left\{\left|P_{j}^{a}\right|, R_{j}^{a}\right\}$ and $\mu_{j}=\theta_{j}=1, \forall j \in T$, Theorem 2 naturally suggests a PTAS - we can use linear search for manipulations on all pairs of targets as shown in Alg. 2, where $e_{i}$ is a unit vector with a single one at position $i$. Theorem 3 shows the approximation guarantee, with a proof similar to Theorem 1, which is included in the full version.

Theorem 3. Alg. 2 returns an additive $\max _{i \in[n]} \frac{2 \eta\left(R_{i}^{d}-P_{i}^{d}\right)}{R_{i}^{a}}$ approximate solution.

\section{Optimizing Payoff with Budget Constraint in Other Forms}

In this section, we explore budget constraints in other forms and show polynomial time algorithms correspondingly.

\subsection{Weighted $L^{\infty}$-norm Form}

When the budget constraint is in weighted $L^{\infty}$-norm form, the defender can make changes to $R^{a}$ and $P^{a}$ for every target to the extent specified by $B_{i}^{r}$ and $B_{i}^{p}$ respectively. Equivalently, the defender can choose $R^{a}$ and $P^{a}$ from a given range. A real-world setting for this problem is when a higher level of authority specifies a range of penalty for activities incurring pollution and allow the local agencies to determine the concrete level of penalty for different activities.

We observe that Condition 2 of Property 1 still holds in this setting. Therefore, such problem can be solved by simply solving $n$ subproblems. In the $i^{\text {th }}$ subproblem which assumes $i$ is the attack target, we may set reward and penalty of $i$ to be the upper bound in the given range and choose the lower bound for other targets. With our improved ORIGAMI-BS algorithm, this problem can be solved in $O\left(n^{2} \log n\right)$ time. 
Theorem 4. With budget constraint in weighted $L^{\infty}$-norm, solving for defender's optimal strategy reduces to solving for defender's optimal coverage in fixed-payoff security games.

\section{2 $L^{0}$-norm Form}

When the budget constraint is in $L^{0}$-norm form, the defender needs to choose which targets to make changes, while the amount of change can be unconstrained. Notice that allowing the defender to arbitrarily modify the attacker's reward $R^{a}$ is not practical and will lead to a trivial solution: the defender will place all coverage on one attack target $t=\arg \max _{i} R_{i}^{d}$ and set $R_{t}^{a}=\infty$. Thus, we only allow the defender to change $P^{a}$ to $\bar{P}^{a}$ and write $\bar{R}^{a}=R^{a}$. This corresponds to the domains where the defender can make some of the targets special. For example, in wildlife protection, legislators can designate some areas as "core zones", where no human activity is allowed and much more severe punishment can be carried out. But the defender cannot set all the areas to be core zones.

We assume the defender has a budget which allows her to change the penalty of $B$ targets. Similar to the $L^{\infty}$ case, we first observe that the defender will choose an extreme penalty value once he decides to change the penalty of a target.

Property 2. There exists an optimal solution where either $\bar{P}_{j}^{a}=-\infty$ for $B$ targets or $\bar{P}_{j}^{a}=-\infty$ for $(B-1)$ targets and $\bar{P}_{t}^{a}=0$ for 1 target. If $\bar{P}_{j}^{a}=-\infty$, then $c_{j}=0$.

Proof. When $t$ is the attack target, the defender would like to maximize $\bar{P}_{t}^{a}$ and minimize $\bar{P}_{j}^{a}$ for all $j \neq t$. If $\bar{P}_{j}^{a}=-\infty$ and $c_{j}>0$, target $j$ will not be attacked as $U_{j}^{a}=-\infty$. In such case, target $j$ is effectively removed from the game.

The defender's problem becomes non-trivial when the budget $B<T$, and we now provide a $O\left(n^{3}\right)$ algorithm (Alg. 3) for solving this problem. We note that several intuitive greedy algorithms do not work, even in more restrictive game settings. A detailed comparison of our algorithm, several greedy algorithms, and a baseline MILP is provided in Section 5.

First, we sort the targets in decreasing attacker's reward $R_{k}^{a}$. Let $E_{k}=\frac{1}{R_{k}^{a}-P_{k}^{a}}$ and $\bar{E}_{k}=\frac{1}{\bar{R}_{k}^{a}-\bar{P}_{k}^{a}}$ for all $k \in T$. When $i$ is the attack target, by Property 2 , we have $\bar{E}_{i} \in\left\{1 / R_{i}^{a}, E_{i}\right\}$ and $\bar{E}_{j} \in\left\{0, E_{j}\right\}$. Let $\Gamma_{l}=\{1,2, \ldots, l\}$ for $l=1,2, \ldots, n$. We notice that one of the $\Gamma_{l}$ 's, denoted as $\Gamma_{l^{*}}$, encapsulates the attack set in the optimal solution to our problem. That is, in the optimal solution, each target in the attack set is in $\Gamma_{l^{*}}$; those targets not in the attack set either are outside $\Gamma_{l^{*}}$, or, if they are in $\Gamma_{l^{*}}$, have $\bar{P}^{a}=-\infty$. A proof can be found in the full version. This allows us to formally define a subproblem $Q_{l, i}$ : assume (i) the optimal attack set is encapsulated by $\Gamma_{l}$, (ii) the attack target is $i \in \Gamma_{l}$, and (iii) no target is covered with certainty, what is the defender's optimal strategy $(c, \bar{E})$ ? A subproblem may be infeasible. First, we show that $Q_{l, i}$ can be solved in $O(n)$ time. From Equation 21, for $Q_{l, i}$, we have

$$
\frac{c_{i}}{\bar{E}_{i}}=\frac{\sum_{k \in \Gamma_{l} \backslash\{i\}}\left(R_{i}^{a}-R_{k}^{a}\right) \bar{E}_{k}+r}{\bar{E}_{i}+\sum_{k \in \Gamma_{l} \backslash\{i\}} \bar{E}_{k}}
$$

Let $s=\min \{B, l-2\}$ if $\bar{E}_{i}=E_{i}$ and $s=\min \{B-$ $1, l-2\}$ if $\bar{E}_{i}=\frac{1}{R_{i}}$. Then $Q_{l, i}$ reduces to finding $s$ out of the $(l-1) \bar{E}_{k}$ 's to set to 0 , and set the rest $\bar{E}_{k}=E_{k}$, so as

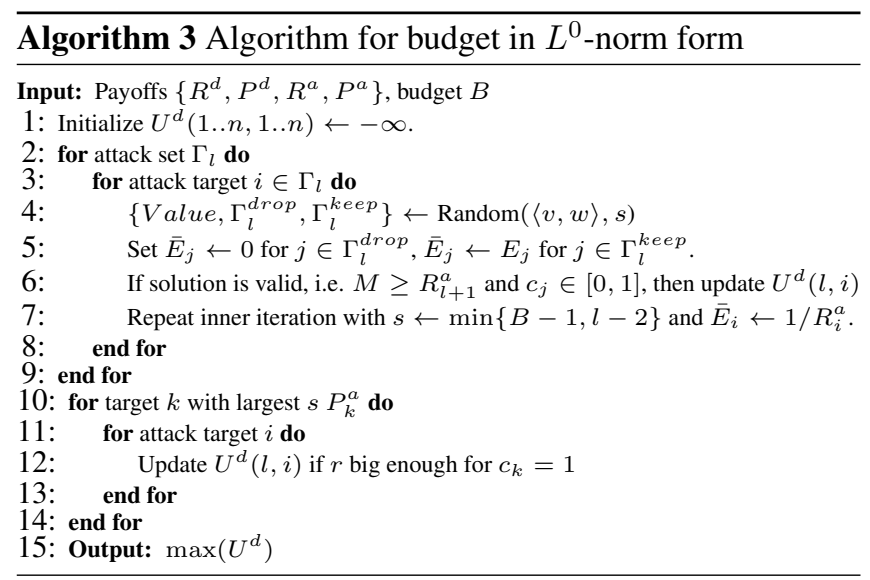

to maximize the above quotient. As a result, $Q_{l, i}$ is closely connected to the problem of choosing subsets with maximum weighted average, which can be solved efficiently.

Proposition 1. [Eppstein and Hirschberg, 1997] Given a set $S$ where $|S|=n$, real numbers $\left\{v_{k}: k \in S\right\}$, positive weights $\left\{w_{k}: k \in S\right\}$, and an integer $r$. Among all subsets of $S$ of order $n-r$, a subset $T \subset S$ which maximizes $A(T)=$ $\frac{\sum_{k \in T} v_{k}}{\sum_{k \in T} w_{k}}$ can be found in $O(n)$ time.

Lemma 1. The subproblem $Q_{l, i}$ can be solved in $O(n)$ time.

Proof. Consider Equation 22. We equate $v_{k}=\left(R_{i}-\right.$ $\left.R_{k}\right) E_{k}+\frac{m}{l-s-1}$ and $w_{k}=E_{k}+\frac{1}{l-s-1} \bar{E}_{i}$. Let $v=\left\{v_{k}\right.$ : $\left.k \in \Gamma_{l} \backslash\{i\}\right\}, w=\left\{w_{k}: k \in \Gamma_{l} \backslash\{i\}\right\}$. By Property 2, we may assume $s$ targets will be removed. Finding a subset $T \subset \Gamma_{l} \backslash\{i\},|T|=l-s-1$, to maximize $A(T)$ is equivalent to our problem to maximize the quotient in Equation 22.

After we find the optimal choices for the $s$ targets, we need to verify on Line 6 of Alg. 3 that the attack set is valid. Since $c_{k}=0$ for $k \notin \Gamma_{l}$, we need $M \geq R_{l+1}^{a}$, where $M$ is the attacker's expected utility as defined in Equation 20. We also need valid coverage probabilities $c_{j}$ 's. These could have been violated by setting some $\bar{P}_{i}$ 's to $-\infty$.

We are now ready to show the main result of this section.

Theorem 5. There is a $O\left(n^{3}\right)$ algorithm for finding the optimal defender strategy with budget constraint in $L^{0}$-norm.

Proof sketch. Consider Alg. 3. Since $R^{a}$ is fixed, $\Gamma_{l}$ 's cover all the attack sets that need to be checked. There are $O\left(n^{2}\right)$ subproblems $Q_{l, i}$. For each $Q_{l, i}$, we run a randomized algorithm for the maximum weighted average problem with expected running time $O(n)$ (Line 4). A deterministic $O(n)$ algorithm exists in [Eppstein and Hirschberg, 1997]. The subproblems $Q_{l, i}$ miss the solutions where some target $j$ is covered with certainty. In this case, $\Gamma_{n}$ is the only possible attack set, and the solution is found on Lines 10-14. A solution is feasible if by removing targets we can keep the sum of coverage probabilities below the defender's resources.

\section{Experimental Results}

\subsection{Simulation Results for $L^{1}$ Budget Problem}

We compare our branch-and-bound (BnB) algorithm (Alg. 1) with three baseline algorithms - NonConv, multiple MILP, 


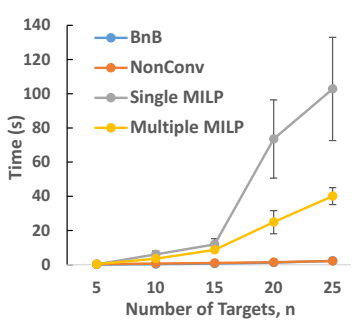

(a) Runtime, small instances

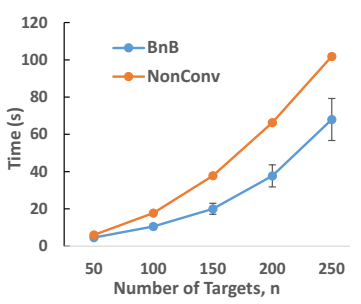

(c) Runtime, large instances

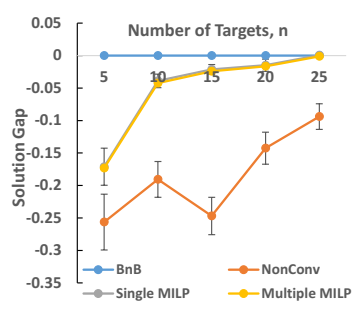

(b) Gap, small instances

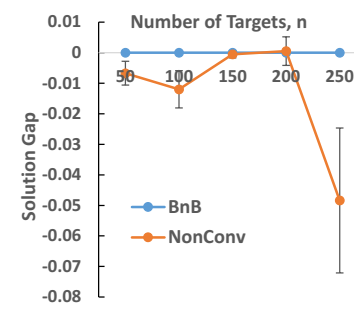

(d) Gap, large instances
Figure 1: Runtime and solution quality for $L^{1}$ case with standard deviation of the mean shown as vertical line

and single MILP. NonConv refers to solving $n$ non-convex optimization problems as shown in Equations 2-8 using IPOPT [Wächter and Biegler, 2006] solver with default parameter setting, which converges to local optima with no global optimality guarantee. Multiple MILPs, as specified by Equations 9-19, and the single MILP formulation, in the full version, are equivalent and have an approximation guarantee specified in Thm. 1. The original payoff structures are randomly generated integers between 1 and $2 n$ with penalties obtained by negation (recall $n$ is the number of targets). Budget and weights of the manipulations are randomly generated integers between 1 and $4 n$.

We set $\rho_{0}=\min _{i \in T} \frac{R_{i}^{a}}{4\left(R_{i}^{d}-P_{i}^{d}\right)}$ which gives an additive $\frac{1}{2}$-approximate solution. Gurobi is used for solving MILPs, which is terminated when either time limit (15 min) or optimality gap (1\%) is achieved. For each problem size, we run 60 experiments on a $\mathrm{PC}$ with Intel Core i7 processor. The solution quality of a particular algorithm is measured by the multiplicative gap between that algorithm and $\mathrm{BnB}$, i.e. $\frac{Z_{\mathrm{A}}-Z_{\mathrm{BnB}}}{Z_{\mathrm{BnB}}}$ where $Z_{\mathrm{A}}$ is best solution value by algorithm $\mathrm{A}$. Thus a positive (negative) gap indicates better (worse) solution value than $\mathrm{BnB}$. We report mean and standard deviation of the mean of runtime and solution quality in Fig. 1. Small instances refer to problem sizes from 5 to 25 . Large instances refer to problem sizes from 50 to 250 .

For problems of small size (Fig. 1a and 1b), BnB finds better solutions in nearly the same time as NonConv, faster than the other two. Since budget size can easily be indivisible by $\rho_{0}$ which is the atomic change we can make, greedy manipulation cannot be achieved by MILPs when such indivisibility happens. On the other hand, BnB first computes a global lower bound using such greedy manipulations, thus creating a gap between BnB and the other two MILP-based algorithms. Indeed the multiplicative gap between the greedy solution and the optimal solution is reported as $0.39 \%$ with a variance of $0.14 \%$. For problems of large size (Fig. 1c and 1d), we only compare BnB and NonConv as the other two algorithms timed out in solving MILP. BnB runs faster than NonConv. It returns better solutions for three problem sizes and nearly the same solution for the other two cases. The MILP-based solution including BnB also has a larger standard deviation in runtime than NonConv.

\subsection{Simulation Results for $L^{0}$ Budget Problem}

We compare the performance of our $O\left(n^{3}\right)$ algorithm with a baseline MILP and two greedy algorithms. Greedy1 removes a target that can lead to most solution quality increase at a time. Greedy 2 starts from the target with highest $\left|P^{d}\right|$ and determines whether to remove it by checking the solution quality before and after removal. Details of these algorithms can be found in the full version.

Initial payoffs are generated in the same way as in the previous subsection. In Fig. 2a, we assume the defender has $r=1$ resource and budget $B=n / 2$, the worst case for the $O\left(n^{3}\right)$ algorithm. The runtime of MILP starts to explode with more than 100 targets, while the $O\left(n^{3}\right)$ algorithm solves the problem rather efficiently. We also note that MILP exhibits high variance in runtime. The variances of other algorithms, including the $O\left(n^{3}\right)$ algorithm, are relatively trivial and thus not plotted. We then test the algorithms with multiple defender resources, as shown in Fig. 2b. With $n$ targets, we assume the defender has $r=n / 10$ units of resources and a budget $B=n / 2$. Most MILP instances reach the time limit of 5 minutes when $n \geq 100$. Yet the $O\left(n^{3}\right)$ algorithm's runtime is almost the same as the single resource case.

Our $O\left(n^{3}\right)$ algorithm and MILP are guaranteed to provide the optimal solution. In contrast, the greedy algorithms exhibit fast runtime but provide no solution guarantee. We measure the solution quality in Fig. $2 \mathrm{c}$ and $2 \mathrm{~d}$ using $\frac{U_{\text {greedy }}^{d}-p}{U_{\text {opt }}^{d}-p}$ where $p=\min _{j} P_{j}^{d}$. Greedy 1 , which runs slightly slower than Greedy2, achieves higher solution quality but both greedy algorithms can lead to a significant loss. In fact, extreme examples exist, as shown in the full version.

\section{Acknowledgments}

The research is initiated with the support of the CAIS summer scholar program.

\section{References}

[Basilico et al., 2016] N. Basilico, A. Lanzi, and M. Monga. A security game model for remote software protection. In ARES, 2016.

[Blocki et al., 2013] Jeremiah Blocki, Nicolas Christin, Anupam Datta, Ariel D. Procaccia, and Arunesh Sinha. Audit games. In IJCAI, 2013.

[Blocki et al., 2015] Jeremiah Blocki, Nicolas Christin, Anupam Datta, Ariel D. Procaccia, and Arunesh Sinha. Audit games with multiple defender resources. In AAAI'15, pages 791-797, 2015.

[Blum et al., 2014] Avrim Blum, Nika Haghtalab, and Ariel D Procaccia. Learning optimal commitment to overcome insecurity. In NIPS, 2014.

[Conitzer and Sandholm, 2006] Vincent Conitzer and Tuomas Sandholm. Computing the optimal strategy to commit to. In $E C$, 2006. 


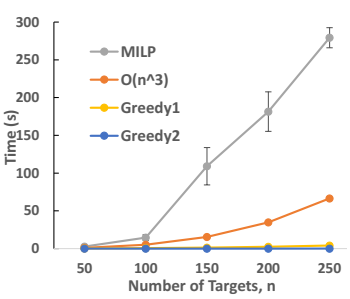

(a) Resource $r=1$

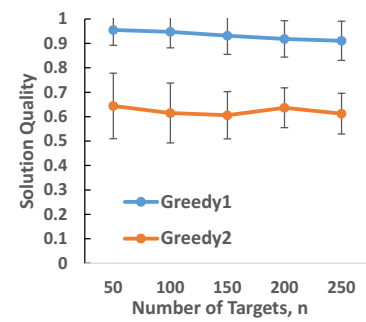

(c) Resource $r=1$

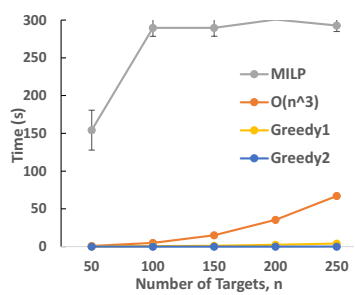

(b) Resource $r=n / 10$

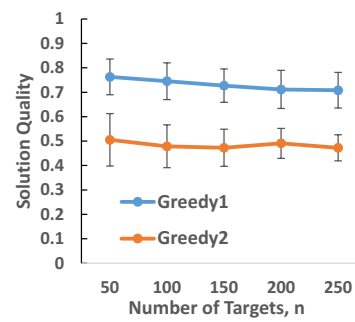

(d) Resource $r=n / 10$
Figure 2: Runtime and solution quality for $L^{0}$ case averaged over 22 trials. MILP has a time limit of 300 seconds. The error bars are standard deviations of the mean.

[Durkota et al., 2015] Karel Durkota, Viliam Lisỳ, Branislav Bosanskỳ, and Christopher Kiekintveld. Optimal network security hardening using attack graph games. In IJCAI, 2015.

[Eppstein and Hirschberg, 1997] David Eppstein and Daniel S. Hirschberg. Choosing subsets with maximum weighted average. J. Algorithms, 24(1):177-193, 1997.

[Fang et al., 2016] Fei Fang, Thanh H. Nguyen, Rob Pickles, Wai Y. Lam, Gopalasamy R. Clements, Bo An, Amandeep Singh, Milind Tambe, and Andrew Lemieux. Deploying paws: Field optimization of the protection assistant for wildlife security. In AAAI, 2016.

[Fujishima et al., 1999] Yuzo Fujishima, Kevin Leyton-Brown, and Yoav Shoham. Taming the computational complexity of combinatorial auctions: Optimal and approximate approaches. In $I J$ CAI, 1999.

[Horák et al., 2017] Karel Horák, Quanyan Zhu, and Branislav Bošanský. Manipulating adversary's belief: A dynamic game approach to deception by design for proactive network security. In GameSec, 2017.

[Kang and Wu, 2015] Xin Kang and Yongdong Wu. Incentive mechanism design for heterogeneous peer-to-peer networks: A stackelberg game approach. IEEE Transactions on Mobile Computing, 14(5):1018-1030, 2015.

[Kiekintveld et al., 2009] Christopher Kiekintveld, Manish Jain, Jason Tsai, James Pita, Fernando Ordóñez, and Milind Tambe. Computing optimal randomized resource allocations for massive security games. In AAMAS '09, pages 689-696, 2009.

[Kiekintveld et al., 2011] Christopher Kiekintveld, Janusz Marecki, and Milind Tambe. Approximation methods for infinite bayesian stackelberg games: Modeling distributional payoff uncertainty. In $A A M A S, 2011$.

[Kiekintveld et al., 2013] Christopher Kiekintveld, Towhidul Islam, and Vladik Kreinovich. Security games with interval uncertainty. In $A A M A S$ '13, 2013.
[Kiekintveld et al., 2015] Christopher Kiekintveld, Viliam Lisỳ, and Radek Píbil. Game-theoretic foundations for the strategic use of honeypots in network security. In Cyber Warfare. 2015.

[Korzhyk et al., 2010] Dmytro Korzhyk, Vincent Conitzer, and Ronald Parr. Complexity of computing optimal stackelberg strategies in security resource allocation games. In $A A A I, 2010$.

[Laszka et al., 2017] Aron Laszka, Yevgeniy Vorobeychik, Daniel Fabbri, Chao Yan, and Bradley Malin. A game-theoretic approach for alert prioritization. In AICS, 2017.

[Letchford and Conitzer, 2013] Joshua Letchford and Vincent Conitzer. Solving security games on graphs via marginal probabilities. In $A A A I, 2013$.

[Letchford et al., 2009] Joshua Letchford, Vincent Conitzer, and Kamesh Munagala. Learning and approximating the optimal strategy to commit to. In Marios Mavronicolas and Vicky G. Papadopoulou, editors, Algorithmic Game Theory, pages 250-262. Springer Berlin Heidelberg, 2009.

[Myerson, 1989] Roger B Myerson. Mechanism design. The New Palgrave: Allocation, Information, and Markets, 1989.

[Paruchuri et al., 2008] Praveen Paruchuri, Jonathan P. Pearce, Janusz Marecki, Milind Tambe, Fernando Ordonez, and Sarit Kraus. Playing games for security: An efficient exact algorithm for solving bayesian stackelberg games. In AAMAS '08, 2008.

[Píbil et al., 2012] Radek Píbil, Viliam Lisỳ, Christopher Kiekintveld, Branislav Bošanskỳ, and Michal Pěchouček. Game theoretic model of strategic honeypot selection in computer networks. In GameSec, 2012.

[Schlenker et al., 2018] Aaron Schlenker, Omkar Thakoor, Haifeng $\mathrm{Xu}$, Milind Tambe, Phebe Vayanos, Fei Fang, Long Tran-Thanh, and Yevgeniy Vorobeychik. Deceiving cyber adversaries: A game theoretic approach. In AAMAS, 2018.

[Sharma and Williamson, 2007] Yogeshwer Sharma and David P. Williamson. Stackelberg thresholds in network routing games or the value of altruism. In EC, 2007.

[Wächter and Biegler, 2006] Andreas Wächter and Lorenz T Biegler. On the implementation of an interior-point filter linesearch algorithm for large-scale nonlinear programming. Mathematical programming, 106(1):25-57, 2006.

[Wang et al., 2016] Zhen Wang, Yue Yin, and Bo An. Computing optimal monitoring strategy for detecting terrorist plots. In $A A A I$, 2016.

[Xue et al., 2016] Yexiang Xue, Ian Davies, Daniel Fink, Christopher Wood, and Carla P. Gomes. Behavior identification in twostage games for incentivizing citizen science exploration. In Principles and Practice of Constraint Programming. Springer, 2016.

[Yang et al., 2012] Dejun Yang, Guoliang Xue, Xi Fang, and Jian Tang. Crowdsourcing to smartphones: Incentive mechanism design for mobile phone sensing. In Mobicom '12, 2012.

[Yin and Tambe, 2012] Zhengyu Yin and Milind Tambe. A unified method for handling discrete and continuous uncertainty in bayesian stackelberg games. In AAMAS '12, 2012.

[Yin et al., 2012] Zhengyu Yin, Albert Xin Jiang, Matthew Paul Johnson, Christopher Kiekintveld, Kevin Leyton-Brown, Tuomas Sandholm, Milind Tambe, and John P Sullivan. Trusts: Scheduling randomized patrols for fare inspection in transit systems. In IAAI, 2012. 